\title{
Contaminación del Aire Atmosférico por Material Particulado en una Ciudad Intermedia: El Caso de Chillán (Chile)
}

\author{
José E. Celis ${ }^{(1) *}$, José R. Morales ${ }^{(2)}$, Claudio A. Zaror ${ }^{(3)}$ y Omar F. Carvacho( ${ }^{4)}$ \\ (1) Universidad de Concepción, Facultad de Medicina Veterinaria, Depto. Ciencias Pecuarias, \\ Casilla 537, Chillán-Chile (e-mail: jcelis@udec.cl) \\ (2) Universidad de Chile, Facultad de Ciencias, Depto. de Física, Casilla 653, Santiago-Chile. \\ (3) Univ. de Concepción, Fac. de Ingeniería, Depto. Ing. Química, Casilla 160-C, Concepción-Chile. \\ (4) University of California, Crocker Nuclear Laboratory, Davis, CA 95616, USA.
}

* autor a quien debe ser dirigida la correspondencia

\section{Resumen}

A través de un análisis de la contaminación urbana de la ciudad de Chillán en Chile, el artículo demuestra que el aumento de la contaminación atmosférica durante otoño e invierno en la ciudad de Chillán debe ser considerado como un problema de origen antropogénico. Esto se explica mayormente debido al uso masivo de la madera como combustible para la calefacción residencial dentro de las áreas urbanas de la ciudad y que se manifiesta a través de una densa nube de humo en los días con estabilidad atmosférica. Esto sugiere que es probablemente necesario un control de la combustión de leña para la calefacción residencial en los días más críticos. Por otra parte, la mayor contaminación en el centro de la ciudad sugiere que este abatimiento se puede alcanzar junto con regulaciones públicas del transporte y de la industria. Sólo así se facilitará el desarrollo sustentable de ciudades intermedias como Chillán, y no repetir los graves problemas de contaminación de las grandes urbes.

Palabras clave: contaminación del aire, medio ambiente, control de emisiones, transporte urbano

\section{Air pollution in a middle-size city: the case of Chillán (Chile)}

\begin{abstract}
Through an analysis of the urban contamination of the city of Chillán in Chile, it is demonstrated that the increase in atmospheric pollution during autumn and winter in the city of Chillán must be considered as a problem of anthropogenic origin. This is explained mainly due to the massive use of wood for residential heating in urban areas of the city and that produces a dense smoke-type cloud during days of stable atmospheric conditions. This suggests that it is probably necessary to control wood combustion for home heating during the most critical days. Also, the increase in pollution in the center of the city indicates that decreasing the pollution level can be also done with government regulations on transport and industry. In this way only, sustainable development of medium size cities as Chillán will be facilitated, not repeating the serious problems of large polluted cities.
\end{abstract}

Keywords: air pollution, environment, emission control, urban transport 


\section{INTRODUCCIÓN}

Las ciudades chilenas muestran claros indicios de deterioro ambiental, según lo han concluido diversos estudios de la Comisión Nacional del Medio Ambiente y de las Universidades que han trabajado los aspectos urbanos, fundamentalmente de las grandes metrópolis y algunas ciudades intermedias (Sandoval, 1999). La mayoría de estos temas dicen relación con graves problemas asociados a la contaminación del aire, agua, suelos, pérdidas de las cubiertas vegetales, carencia de áreas verdes urbanas, profunda segregación social de sus habitantes, entre otros (Azócar et al., 2003). Las áreas metropolitanas del territorio nacional, como Santiago y Concepción-Talcahuano, han debido ser declaradas ambientalmente saturadas debido a que sus medios naturales ya no son capaces de reciclar o disipar las enormes cantidades de desechos domésticos, vehiculares e industriales que emiten a la atmósfera y que contaminan también el suelo y los cuerpos de agua. Lo grave es que este proceso de deterioro conduce a una pérdida de la calidad de vida de los habitantes de la mayoría de las ciudades de la región latinoamericana, que se agudiza por la continua migración desde el campo hacia sectores urbanos.

Uno de los aspectos menos estudiados se refiere a la contaminación aérea en ciudades intermedias latinoamericanas. Se sabe que la contaminación aérea por material particulado afecta negativamente la calidad del aire y la salud de las personas (Sánchez y Morel, 1995). De hecho, tal como lo señalan las investigaciones, esta contaminación en las ciudades está ligada directamente a las actividades antropogénicas, donde se conjugan el transporte vehicular, la actividad industrial, el movimiento de las personas, los espacios con poca ventilación, y el microclima característico de la urbe (Querol et al., 2001).

La presencia de la ciudad modifica las condiciones atmosféricas naturales. Las construcciones alteran la rugosidad de la superficie, la humedad del suelo y el balance térmico en la superficie, lo cual trae aparejado una capa atmosférica urbana que suele ser más seca y cálida que el entorno (Ulriksen, 1993). Este aumento de la temperatura dentro de la ciudad con respecto al entorno (isla térmica), se manifiesta de preferencia en las noches despejadas de invierno. La isla térmica que se produce en Santiago en algunas noches es $10^{\circ} \mathrm{C}$ mayor que el entorno suburbano (Salinas, 1982). El efecto de isla térmica tiende a elevar la capa de mezcla sobre la ciudad por sobre el entorno, debilitando la ventilación natural. A esto se suma la mayor rugosidad superficial en el centro de las ciudades, todo lo cual frena el flujo de aire a nivel superficial, que es donde viven las personas (Ulriksen, 1993). Esta situación ha obligado a realizar estudios en las grandes ciudades, que han permitido predecir la calidad del aire ante ciertos eventos atmosféricos.

En Latinoamérica hay pocos estudios sobre contaminación por aerosoles atmosféricos, aunque los resultados permiten concluir que los problemas son los mismos que se observan en los países desarrollados (Romieu y Borja-Aburto, 1997; Castaño y Artaxo, 2001; Bogo et al., 2003). Sin embargo, la determinación de los aerosoles es cara y laboriosa, y los resultados no son aplicables a otras ciudades. En el caso de Chile, el mayor énfasis está dado en la determinación de estos contaminantes en las grandes ciudades como Santiago, por el enorme impacto que tienen sobre la salud de las personas, donde cohabita el $35 \%$ de la población chilena, y cuyo emplazamiento no le brinda una adecuada ventilación natural (Sandoval, 1999; Kavouras et al., 1999; Tsapakis et al., 2002). Los estudios señalan que en Santiago (que ha llegado hasta los $140 \mu \mathrm{g} / \mathrm{m}^{3}$ ) una reducción de $10 \mu \mathrm{g} / \mathrm{m}^{3}$ de $\mathrm{PM}_{10}$ producirá una disminución del orden del 3 al $7 \%$ en las consultas médicas, con todos los beneficios asociados por menores costos en consultas médicas, hospitalizaciones y ausentismo laboral (Sánchez y Morel, 1995). Esto ha ayudado ha implementar un moderno plan de transporte urbano, que sin duda ayudará a aliviar el problema de la contaminación aérea en Santiago. Por otro lado, un estudio llevado a cabo en cinco ciudades chilenas, Iquique, Valparaíso, Viña del Mar, Rancagua y Temuco, reveló que en la mayoría de las ciudades las concentraciones del $\mathrm{PM}_{10}$ fueron entre un 11 a un $55 \%$ mayores que los estándares estadounidenses (Kavouras et al., 2001).

Pese a todo, muy poco esfuerzo se ha realizado en la medición de los aerosoles atmosféricos en ciudades intermedias como Chillán, donde no se sabe el impacto que tienen. La ciudad de Chillán es un núcleo administrativo que ejerce una fuerte atracción sobre los asentamientos urbanos de la 
provincia de Ñuble y sobre el entorno agrario circundante, prestando servicios y constituyendo un foco de atracción para el asentamiento de la población (Azócar et al., 2003). Estas ciudades entre 50.000 y 250.000 habitantes, están creciendo aceleradamente y son las que presentan mayores expectativas de desarrollo sustentable, con claros indicios de reconversión agrícola a semi-industrial. Es necesario evitar que estas ciudades reproduzcan los problemas ambientales de las áreas metropolitanas, por lo que es importante evaluar rápida y estratégicamente sus potencialidades y limitaciones ambientales. Las autoridades tendrán necesariamente que adoptar medidas para reducir la contaminación aérea a lo largo de todo el territorio nacional, de acuerdo a las normas que regirán a los países que quieran pertenecer al mundo globalizado, y deberán contar con información al respecto.

Como consecuencia, el propósito de este estudio fue determinar la contaminación por material particulado del aire respirable en la atmósfera de la ciudad de Chillán, y al mismo tiempo estudiar la variación temporal y espacial del polvo fugitivo. A través del análisis de los estudios que conectan diferentes niveles de concentración tóxica con problemas de salud, el artículo se propone demostrar que Chillán presenta características morfológicas y tráfico muy particulares que hacen suponer que el material particulado en el área central de la ciudad proviene principalmente de fuentes móviles (transporte urbano). Por el contrario, en los suburbios de la ciudad los aerosoles provienen de fuentes fijas diversas (residencias, talleres, y actividades agrícolas que rodean la ciudad). Con ello se espera aportar conocimiento acerca de la calidad del aire respirable que sirva para complementar la información disponible a nivel nacional.

\section{EXPERIMENTAL}

\section{Ubicación y descripción del área}

La ciudad de Chillán se localiza en el extremo nor-occidental de la cuenca del río Chillán, que a su vez se ubica en el sector nor-central de la región del Biobío, en $36^{\circ} 34^{\prime}$ latitud Sur, $72^{\circ} 06^{\prime}$ longitud Oeste, y a 144 m.s.n.m. Según el último Censo 2002, la Región del Biobío $(36.929,3$ km²) cuenta con una población estimada de 1.861 .562 habitantes, con una densidad de $51,7 \mathrm{hab} / \mathrm{km}^{2}$ que supera a la media nacional $\left(20 \mathrm{hab} / \mathrm{km}^{2}\right)$, concentra su población urbana en tres grandes polos: Concepción (con 900.000 habitantes aprox.), Chillán (con 250.000 personas aprox.), y Los Angeles (con aprox. 200.000 habitantes). En ellas se concentra el $73 \%$ de la población regional.

\section{Cuantificación del parque vehicular}

Se identificó la arteria urbana con mayor tráfico vehicular, y se determinó el flujo vehicular como la cantidad de vehículos que circulan por hora. Las fuentes móviles de la ciudad de Chillán están constituidas por microbuses, taxis, colectivos, camiones y automóviles particulares. El parque vehicular fue obtenido a partir del registro proporcionado por la Municipalidad de Chillán en el año 2002, identificando autos particulares, colectivos, taxis, microbuses y camiones.

\section{Cuantificación del parque industrial}

Para ello se utilizaron fotografías aéreas, se clasificaron las industrias por tipo de actividad (agricultura, construcción, transporte, etc.), recorridos en terreno para posicionar con GPS cada una de las industrias, SIG (ArcView 3.2 y Arc-Info N.T.), y la información generada por Azócar et al. (2003). Las emisiones del sector industrial fueron identificadas a partir de la información de los combustibles utilizados en calderas y hornos industriales.

\section{Cuantificación del polvo fugitivo}

Las concentraciones de material particulado respirable se determinaron con monitores IMPROVE equipados con cabezal Sierra Andersen modelo 246b para partículas menores a $10 \mu \mathrm{m}$, ubicado a 3 $\mathrm{m}$ de altura, según el estándar de la EPA. Se usaron filtros de teflón de $25 \mathrm{~mm}$ de diámetro y de fibra de vidrio de $47 \mathrm{~mm}$. Se ubicaron seis monitores en la ciudad de Chillán, considerando para ello las fuentes fijas y móviles, así como el centro de la ciudad, y las industrias. También se tomaron en 
cuenta lugares de valor social, como ser la zona de hospital, y se consideró la dirección de los vientos predominantes (SW en primavera-verano, y NE en otoño-invierno). La configuración de los sitios de monitoreo es la que se muestra en la Fig. 1. En cada punto de muestreo, el programa de monitoreo permitió colectar material particulado $\mathrm{PM}_{10}$.

La concentración del $\mathrm{PM}_{10}$ se determinó usando una microbalanza Cahn 31, pesando los filtros antes y después de cada monitoreo. El tiempo de muestreo de cada filtro fue de 24 horas para el periodo comprendido entre el 1 Septiembre del 2001 al 31 Diciembre del 2003. Con el propósito de estudiar la variación del material particulado en los días laborales y fines de semana, durante el mes de febrero 2003 el sitio de muestreo $\mathrm{M}_{\mathrm{c}}$ se destinó exclusivamente a recolectar filtros para determinar su concentración entre lunes a viernes, y entre sábado y domingo.

Para la determinación de los compuestos químicos contenidos en los filtros, se usaron las técnicas de laboratorio PIXE y XRF, en los laboratorios del Crocker Nuclear Laboratory de la University of California-Davis, cuya descripción se encuentra en la literatura (Brook et al., 1997). También, en el laboratorio de Recursos Renovables de la Universidad de Concepción, se determinó carbono orgánico (CO) a través de un analizador termal óptico DRI modelo 4000X. La concentración de la materia orgánica (MO) se determinó multiplicando por 1,4 el contenido de CO (Kim et al., 2000). El carbono elemental (CE) se determinó usando el método termográfico descrito por Röösli et al. (2001). El CO y el CE son importantes pues influencian las diferentes reacciones de partículas y gases que ocurren en la atmósfera (Viidanoja et al., 2002). La detección de amonio $\left(\mathrm{NH}_{4}{ }^{+}\right)$se realizó por medio de un espectrómetro Lambda 2 Perkin Elmer. De acuerdo a lo recomendado por Chow y Watson (1999), los iones nitrato $\left(\mathrm{NO}_{3}{ }^{-}\right)$y sulfato $\left(\mathrm{SO}_{4}{ }^{2-}\right)$ fueron determinados por cromatografía de iones, usando para ello un equipo Dionex DX 100.

\section{RESULTADOS Y DISCUSION}

\section{Características de la ciudad}

La ciudad de Chillán se localiza en la depresión central, definida como una llanura de depósitos fluviales y fluviovolcánicos, caracterizada por una topografía plana a ligeramente ondulada, con pendientes suaves (Borgel, 1986). Estos depósitos fueron transportados desde la Cordillera de Los Andes por los ríos Chillán y Ñuble, como consecuencia de los grandes eventos volcánicos y torrenciales. De acuerdo con el último censo realizado en el año 2002, su población es de 162.930 habitantes, y de acuerdo con los pocos estudios que se han desarrollado, es una de las ciudades chilenas intermedias que han experimentado un significativo crecimiento urbano en las últimas décadas, donde Chillán ha tenido un crecimiento poblacional de 2,2 veces en los últimos 50 años.

Respecto del uso del suelo, Olave (1996) estima que la ciudad está caracterizada fundamentalmente por presentar una construcción continua. La dinámica de la población de Chillán ha influenciado el crecimiento del área urbana, especialmente en lo relativo al uso residencial y de servicios. Este extraordinario crecimiento urbano se manifiesta también en la construcción de poblaciones pertenecientes a todos los estratos sociales. Con posterioridad a 1992 este proceso se aceleró, como lo demuestra la construcción de varias poblaciones catalogadas como pobres y de medianos ingresos. Para la población de altos ingresos se han construido áreas caracterizadas como condominios cerrados, con hermosas casas de material sólido de más de $150 \mathrm{~m}^{2}$ construidos.

La economía de la región basa su desarrollo en actividades agrícola, forestal, ganadera y turística. En la ciudad de Chillán se concentran el comercio, las actividades financieras, construcción, transporte, comunicaciones y la industria del aserrío de la madera. La ciudad presenta un damero central cuadrado de 168 hectáreas, al estilo de los típicos pueblos de la colonia, enmarcada por las calles Ecuador, Argentina, Collín y Brasil. La superficie urbanizada de la ciudad de Chillán es de aproximadamente 2.010 hectáreas y la superficie de áreas verdes es de 243 hectáreas. Sin embargo, en 1998 Chillán presentaba un $60 \%$ de calles sin pavimentar (PLADECO, 1998).

En la Fig. 2 están representadas todas las fuentes fijas industriales de la ciudad. Esta información permitió identificar los tipos de industrias y su ubicación en el área estudiada, con lo cual se pudieron 
hacer muestreos precisos. Si bien no existe un parque industrial de grandes dimensiones, y casi la mayoría son del rubro de aserrío de maderas, se observa que la mayor concentración de ellas se ubica en el sector surponiente de Chillán, justamente donde los vientos soplan con dirección suroriente, llevando los contaminantes hacia la ciudad. Esto debiera llevar a las autoridades locales a implementar un efectivo control de emisiones.

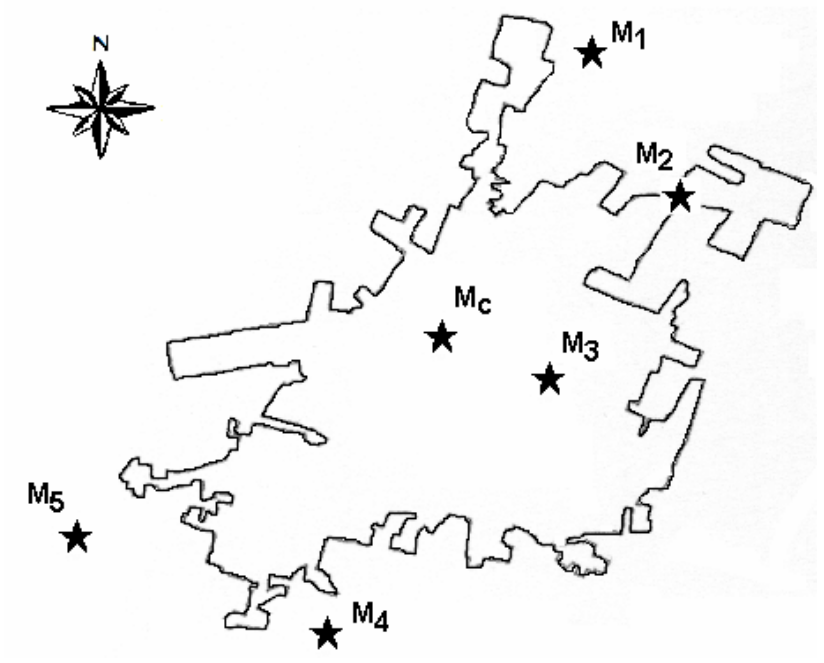

Fig. 1: Ubicación de los monitores en Chillán.

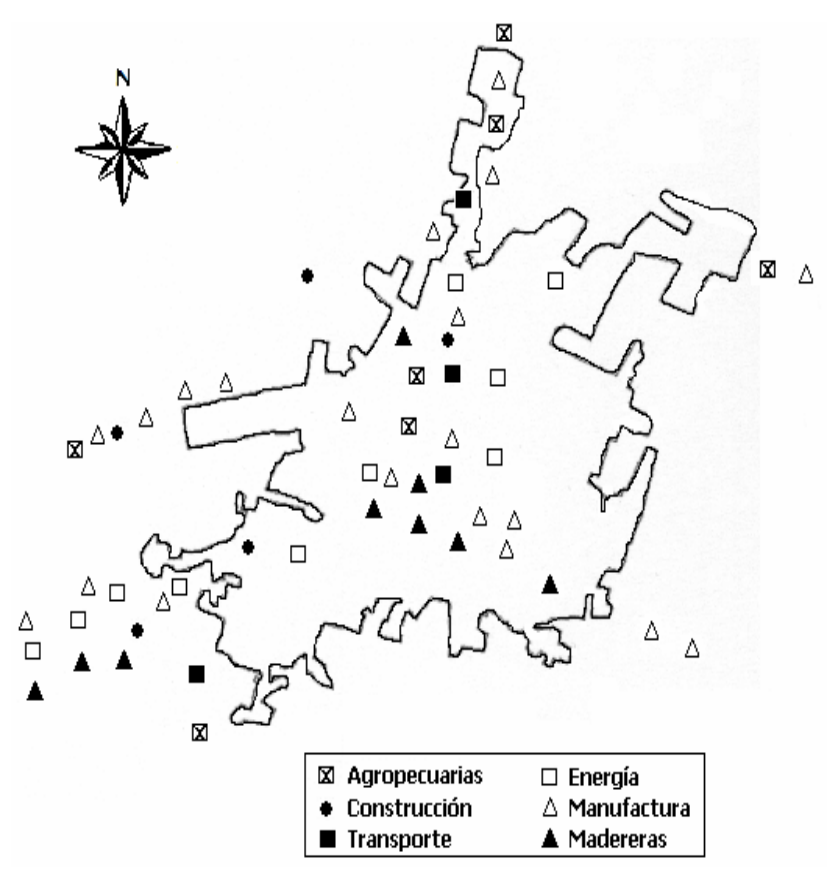

Fig. 2: Tipo y ubicación de las industrias en la ciudad de Chillán.

Dentro de las fuentes fijas, tiene gran importancia la calefacción domiciliaria. La ciudad de Chillán representa un centro poblacional, en el que las prácticas comunes del uso de la madera como combustible para la calefacción en los meses de invierno, agrega otro factor que afecta la calidad del aire respirable. Las emisiones del sector industrial, que corresponden en su mayoría a madereras, agroindustrias y panaderías, proceden principalmente de los combustibles utilizados en calderas y hornos industriales, en los cuales mayoritariamente se usa leña y/o petróleo. Con relación a las fuentes móviles, la ciudad de Chillán tiene uno de los mayores parques de locomoción colectiva en proporción a su población. Las fuentes móviles de la ciudad de Chillán están constituidas por microbuses, taxis, colectivos, camiones y automóviles particulares. Según datos proporcionados por la Municipalidad de Chillán, en el año 2002 el parque vehicular tenía 19.428 vehículos. De estos, 16.677 son autos particulares, 464 son colectivos, 329 son taxis, 325 son microbuses, y 1.633 son camiones. A la vez, resulta muy contaminante, pues se trata en su mayoría de vehículos que han sido retirados de circulación en Santiago. Otra característica es que todo el transporte colectivo en Chillán circula obstinadamente (y permisiblemente) por el centro urbano de la ciudad. El estudio realizado confirmó que circulan entre 1000 a 1400 máquinas del transporte público cada hora, en calle 5 de Abril (entre Maipón y El Roble), de las cuales un $60 \%$ son taxis colectivos y un $20 \%$ taxibuses. Del total de taxibuses existentes, el 95\% tiene más de 10 años de uso.

\section{Contaminación del aire}

En la Tabla 1 están indicadas las concentraciones de $\mathrm{PM}_{10}$ promedio anual en cada una de las estaciones de monitoreo.

Tabla 1: Concentraciones de PM10 promedio anual $\left(\mu \mathrm{g} / \mathrm{m}^{3}\right)$ para cada estación de monitoreo en la ciudad de Chillán entre el 1 de enero al 31 de diciembre 2003.

\begin{tabular}{|c|c|c|c|c|c|c|}
\hline $\mathrm{M}_{1}$ & $\mathrm{M}_{2}$ & $\mathrm{M}_{3}$ & $\mathrm{M}_{4}$ & $\mathrm{M}_{5}$ & $\mathrm{M}_{\mathrm{c}}$ & Media \\
\hline $43,8 \pm 9,3$ & $59,5 \pm 1,2$ & $76,6 \pm 3,4$ & $53,7 \pm 0,5$ & $64,3 \pm 0,3$ & $83,4 \pm 12,8$ & $63,5 \pm 14,6$ \\
\hline
\end{tabular}




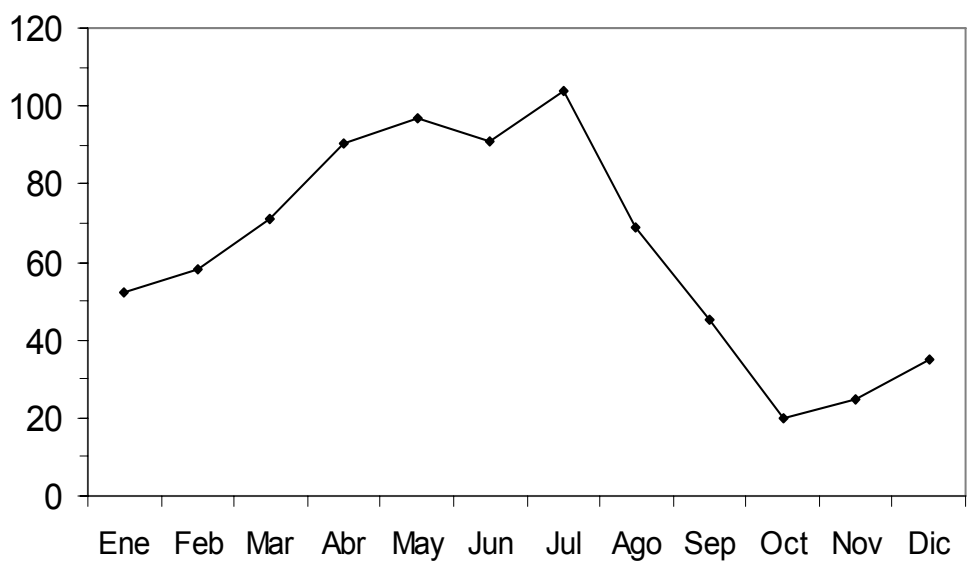

Fig. 3: Concentración promedio del material particulado $\mathrm{PM}_{10}(\mu \mathrm{g} / \mathrm{m} 3)$, periodo 2001-2003.

Se puede apreciar que en la periferia de la ciudad de Chillán $\left(\mathrm{M}_{1}\right)$ la concentración de aerosoles fue un $47,5 \%$ menor que en el centro $\left(M_{c}\right)$. En general, considerando los seis sitios de monitoreo, la concentración de $\mathrm{PM}_{10}$ promedio anual para Chillán $\left(63,5 \pm 14,6 \mu \mathrm{g} / \mathrm{m}^{3}\right)$ fue un $27 \%$ mayor que la norma de $50 \mu \mathrm{g} / \mathrm{m}^{3}$, establecida en el D.S. 45/01 del Ministerio Secretaría General de la Presidencia de la República, y que entró en aplicación en enero del 2002.

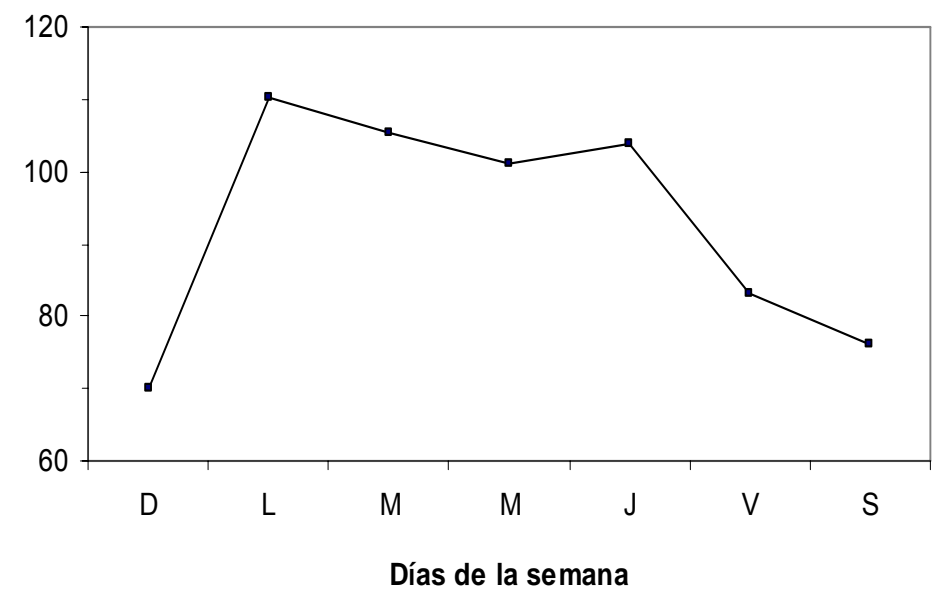

Fig. 4: $\mathrm{PM}_{10}\left(\mu \mathrm{g} / \mathrm{m}^{3}\right)$ promedio semanal en el centro de la ciudad de Chillán (Febrero 2003).

Según Kavouras et al. (2001), es inferior a lo medido en Temuco $\left(67,7 \pm 3,2 \mu \mathrm{g} / \mathrm{m}^{3}\right)$, Rancagua $(73,8$ $\left.\pm 4,2 \mu \mathrm{g} / \mathrm{m}^{3}\right)$, Valparaíso $\left(77,5 \pm 2,7 \mu \mathrm{g} / \mathrm{m}^{3}\right)$, pero supera a lo registrado en lquique $\left(62,1 \pm 3,5 \mu \mathrm{g} / \mathrm{m}^{3}\right)$ y Viña del Mar $\left(55,5 \pm 3,5 \mu \mathrm{g} / \mathrm{m}^{3}\right)$. También es inferior a Ciudad de México $\left(115 \mu \mathrm{g} / \mathrm{m}^{3}\right)$. Según Brook et al. (1997), supera las concentraciones registradas en ciudades canadienses como Montreal $(27,8$ $\left.\mu \mathrm{g} / \mathrm{m}^{3}\right)$, Toronto $\left(28,1 \mu \mathrm{g} / \mathrm{m}^{3}\right)$, Vancouver $\left(26,9 \mu \mathrm{g} / \mathrm{m}^{3}\right)$, y Ottawa $\left(22,6 \mu \mathrm{g} / \mathrm{m}^{3}\right)$. También, es mayor que las mediciones realizadas en Boston $\left(19 \mu \mathrm{g} / \mathrm{m}^{3}\right)$, Washington DC $\left(28 \mu \mathrm{g} / \mathrm{m}^{3}\right)$, Memphis $(30,9$ $\mu \mathrm{g} / \mathrm{m}^{3}$ ), y los $26 \mu \mathrm{g} / \mathrm{m}^{3}$ de Filadelfia (Parkhurst et al., 1997). En Basilea (Suiza) se han registrado concentraciones de aerosoles de $10,8 \mu \mathrm{g} / \mathrm{m}^{3}$ en zonas rurales y $29,6 \mu \mathrm{g} / \mathrm{m}^{3}$ en áreas urbanas (Röösli et al., 2001).

Con relación a la variabilidad temporal de la contaminación aérea de la ciudad de Chillán, en la Fig. 3 puede verse que las mayores concentraciones de material particulado se producen en los meses de invierno, en directa concordancia con lo señalado en la literatura (Préndez y Ulriksen, 1993; Röösli et al., 2001), lo cual en el caso de Chillán se explica por la gran cantidad de leña que se consume (alrededor de $200 \mathrm{mil} \mathrm{m}^{3} /$ año). 
El $\mathrm{PM}_{10}$ fue un $40 \%$ mayor en los días laborales (lunes a viernes) que durante los fines de semana (Fig. 4), reflejando la influencia de la actividad antrópica, como el tráfico, las faenas de construcción y los procesos industriales, los que generalmente son mayores en los días hábiles. Esto es similar a lo encontrado por Röösli et al. (2001), quienes determinaron un 54\% de incremento del $\mathrm{PM}_{10}$ en los días de trabajo respecto de los fines de semana. Probablemente, el tráfico de la ciudad contribuye fuertemente a esta variación, a través del levantamiento de polvo de las calles y la eliminación de partículas finas por el escape de los motores de combustión.

En la Fig. 5 se muestra la composición química del material particulado para la ciudad de Chillán en el periodo estudiado. La materia orgánica (MO) alcanzó el $31 \%$ del $\mathrm{PM}_{10}$, seguido por el material geológico (Suelo) con $27 \%$, el nitrato $\left(\mathrm{NO}_{3}{ }^{-}\right)$con $11 \%$, el carbono elemental (CE) con $8 \%$, el amonio con $\left(\mathrm{NH}_{4}^{+}\right) 7 \%$, y el sulfato $\left(\mathrm{SO}_{4}{ }^{2-}\right)$ con $5 \%$. Los compuestos orgánicos representan la fracción más grande del material particulado, pudiendo llegar hasta el $40 \%$ (Chow et al., 1994). Las posibles fuentes de materia orgánica son los incendios de bosques, las quemas de rastrojos agrícolas, la incineración, la calefacción domiciliaria, la quema de basuras municipales, las emisiones industriales, los residuos de aceites y de petróleo, la combustión de energéticos fósiles, y las emisiones de los crematorios (Celis et al., 2003).

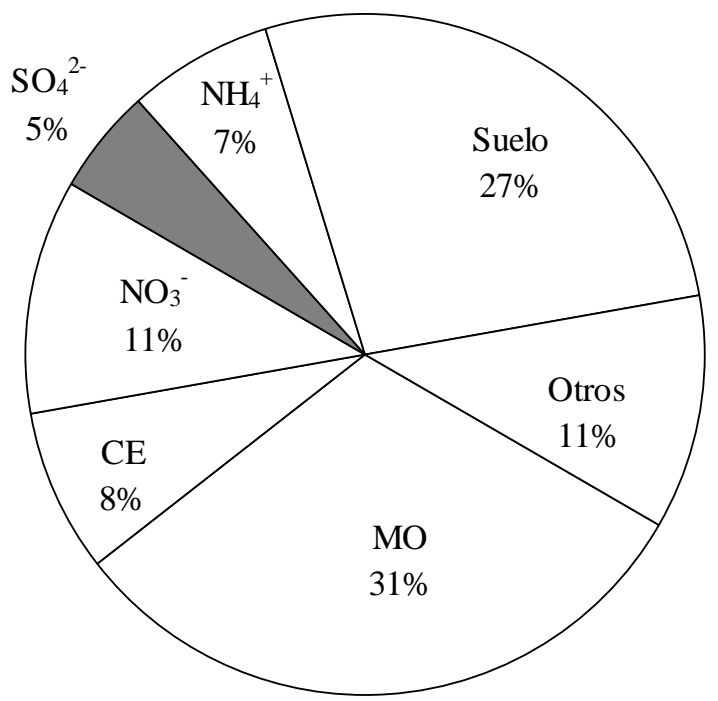

Fig. 5: Composición química del $\mathrm{PM}_{10}$ en la ciudad de Chillán (sep. 2001 a sep. 2002).

En la Tabla 2 se aprecia que la materia orgánica (MO) fue mayor durante los meses fríos que en los meses de calor. Este mismo patrón estacional se presentó para los compuestos $\mathrm{NO}_{3}{ }^{-}$y $\mathrm{NH}_{4}{ }^{+}$. En cambio las mayores proporciones de Suelo se registraron en los meses de más calor, que coincide con una menor precipitación y humedad ambiental. Estos resultados concuerdan con aquellos reportados en otros estudios latinoamericanos (Castaño y Artaxo, 2001; Bogo et al., 2003), sugiriendo con ello que la contaminación aérea durante la estación fría es de origen antropogénico, mientras que en la época de calor es producida principalmente por fuentes naturales.

Las altas concentraciones de Suelo en los meses de calor se deben a la resuspensión del polvo de los caminos y tierras agrícolas que rodean la ciudad de Chillán, La mayoría de los suelos del Valle Central de Chile fueron formados de cenizas volcánicas, y cuando están secos liberan fácilmente muchas partículas por acción del viento y actividades agrícolas (Celis et al,, 2003), A lo anterior se agrega el hecho de que cerca del $60 \%$ de las calles de Chillán aún permanecen sin pavimentar (PLADECO, 1998).

Las formas de crecimiento urbano de la ciudad de Chillán, asociada a factores sociales, económicos y políticos, han contribuido a modelar una ciudad con graves problemas ambientales, En este sentido, Azócar et al, (2003) hablan de tres ciudades en una, con claras diferencias sociales, donde se distinguen los enclaves industriales en los accesos norte y sur, un damero central característico y 
con islotes de ruralidad dentro de los límites urbanos, Lo que está claro, es que la calidad de vida de la ciudad está siendo afectada, razón por la cual las autoridades pronto deberán tomar las providencias que la situación amerita para revertirla, donde sin lugar a dudas el tema de las regulaciones medioambientales será clave para permitir que la ciudad sobrelleve con armonía su crecimiento urbano.

Tabla 2. Promedio estacional de los compuestos químicos del $\mathrm{PM}_{10}\left(\mu \mathrm{g} / \mathrm{m}^{3}\right)$ en la atmósfera de la ciudad de Chillán y datos meteorológicos asociados (Septiembre 2001 y Septiembre 2003).

\begin{tabular}{lrrrr}
\hline & $\begin{array}{c}\text { Primavera } \\
\text { 22 Sep-21 Dic }\end{array}$ & $\begin{array}{c}\text { Verano } \\
\text { 22 Dic - 21 Mar }\end{array}$ & $\begin{array}{c}\text { Otoño } \\
\text { 22 }\end{array}$ & \multicolumn{1}{c}{$\begin{array}{c}\text { Invierno }-21 \text { Jun } \\
\text { 22 Jun - 21 Sep }\end{array}$} \\
\hline & & & & \\
$\mathrm{MO}$ & $14,50 \pm 9,02$ & $14,94 \pm 10,72$ & $39,88 \pm 16,46$ & $33,25 \pm 20,11$ \\
$\mathrm{NO}_{3}{ }^{-}$ & $4,13 \pm 2,13$ & $5,13 \pm 1,99$ & $13,13 \pm 6,03$ & $8,94 \pm 7,43$ \\
$\mathrm{EC}$ & $5,25 \pm 2,73$ & $4,86 \pm 1,87$ & $5,93 \pm 2,05$ & $6,69 \pm 2,07$ \\
$\mathrm{NH}_{4}{ }^{+}$ & $3,66 \pm 1,17$ & $3,11 \pm 1,12$ & $6,40 \pm 1,58$ & $7,18 \pm 2,23$ \\
$\mathrm{SO}_{4}{ }^{2-}$ & $2,14 \pm 0,44$ & $1,60 \pm 1,03$ & $3,21 \pm 1,86$ & $3,51 \pm 2,03$ \\
Suelo $_{\text {Otros }}^{12,31 \pm 2,43}$ & $32,75 \pm 4,31$ & $7,50 \pm 2,47$ & $4,06 \pm 1,78$ \\
Lluvia (mm) & $7,59 \pm 2,22$ & $10,81 \pm 3,31$ & $3,40 \pm 1,11$ & $4,16 \pm 1,28$ \\
Hdad. Rel. (\%) & $253,2 \pm 15,3$ & $130,2 \pm 14,8$ & $468,9 \pm 11,4$ & $570,1 \pm 10,5$ \\
Temp. ( $\left.{ }^{\circ} \mathrm{C}\right)$ & $71,9 \pm 3,2$ & $61,4 \pm 3,7$ & $81,2 \pm 4,2$ & $84,3 \pm 3,7$ \\
\hline
\end{tabular}

\section{CONCLUSIONES}

El artículo demuestra que la contaminación aérea urbana de Chillán es preocupante. Desde el punto de vista temporal, la contaminación del aire atmosférico en la ciudad de Chillán mostró un perfil cíclico claramente definido, con niveles más altos en la estación fría (marzo-agosto). Desde el punto de vista espacial, los niveles de contaminación fueron más altos en el centro urbano de Chillán con respecto a las zonas límites rurales que rodean la ciudad. Los resultados mostraron que en la periferia de Chillán la concentración de aerosoles fue un $47,5 \%$ menor que en el centro de la ciudad.

Durante la estación fría, las sustancias carbonosas y las sustancias inorgánicas de origen secundario fueron los componentes predominantes del aire respirable en Chillán. Por el contrario, durante los meses de calor fueron las partículas de polvo del suelo las que abundaron en la atmósfera de la ciudad. La concentración del $\mathrm{PM}_{10}$ promedio anual para Chillán $\left(63,5 \pm 14,6 \mu \mathrm{g} / \mathrm{m}^{3}\right)$ fue un $27 \%$ mayor que la norma EPA y nacional de $50 \mu \mathrm{g} / \mathrm{m}^{3}$. Comparando con otras ciudades chilenas, es inferior a lo medido en Santiago, Temuco, Rancagua, y Valparaíso, pero supera a lo registrado en lquique y Viña del Mar. Las mayores concentraciones de aerosoles $\mathrm{PM}_{10}$ durante días laborables comparado con los fines de semana, registradas en el centro de la ciudad, son posiblemente la consecuencia de actividades humanas tales como el tráfico de la ciudad, trabajos de construcción, talleres y operaciones industriales.

El damero urbano central de la ciudad de Chillán, enmarcado por las calles Ecuador, Brasil, Collín y Argentina, se caracteriza por un tráfico intenso y por presentar la totalidad de sus calles pavimentadas, por lo que el material particulado en esta área de la ciudad proviene principalmente de fuentes móviles (transporte urbano). En cambio, en los suburbios de la ciudad, con menor tráfico vehicular y un gran número de calles sin pavimentar, los aerosoles provienen de fuentes fijas diversas (residencias, partículas provenientes del polvo de las calles, talleres, y actividades agrícolas que rodean la ciudad).

Los resultados, aunque de cobertura limitada, indican que la atmósfera respirable en la ciudad de Chillán debe ser considerada como un problema de origen antropogénico durante otoño e invierno. Esto se explica mayormente debido al uso masivo de la madera como combustible para la 
calefacción residencial dentro de las áreas urbanas de la ciudad, que se manifiesta a través de una densa nube de humo en los días con estabilidad atmosférica. Esto admite la posibilidad de un control de la combustión de leña para la calefacción residencial en los días con baja temperatura, baja velocidad del viento y alta humedad relativa. Por otra parte, las mayores concentraciones de $\mathrm{PM}_{10}$ medidas en el centro de la ciudad sugieren que este abatimiento se puede alcanzar junto con regulaciones públicas del transporte y de la industria, tales como definir nuevas maneras de evitar tráfico vehicular a través del centro de la ciudad, y prohibiendo el uso de máquinas antiguas que han sido retiradas de circulación en Santiago. Sólo así se facilitará el desarrollo sustentable de ciudades intermedias como Chillán, y no repetir los graves problemas de contaminación de las grandes urbes acompañado de muertes y enfermedades.

La experiencia obtenida de este estudio permite sugerir que en futuros trabajos de calidad del aire urbana en Chillán, deben realizarse correlaciones con salud de la población y análisis multidisciplinarios bio-médicos. Asimismo, los muestreos deben ser más frecuentes, tal vez en intervalos de 3 a 4 horas, para así seguir el comportamiento de las actividades de la ciudad y de las variables meteorológicas. Además, se debe discriminar por tamaño de partículas, especialmente en el sector céntrico de la ciudad de Chillán.

\section{AGRADECIMIENTOS}

Los autores agradecen a la Dirección de Investigación de la Universidad de Concepción, Chile, por el financiamiento otorgado al proyecto 200,132,004-1.1.

\section{REFERENCIAS}

Azócar, G., R. Sanhueza y C. Henríquez, Cambio en los patrones de crecimiento en una ciudad intermedia: el caso de Chillán en Chile Central. EURE 29, 79-82 (2003).

Bogo, H., M.Otero, P.Castro, M. J. Ozafrán, A. Kreiner, E.J. Calvo y R. Martín Negri, Study of atmospheric particulate matter in Buenos Aires city. Atmospheric Environment 37, 1135-1147 (2003).

Borgel, O., Correlaciones entre sismo tectónica y escurrimiento de ríos en crecidas invernales. Revista Geográfica de Chile 29, 41-47 (1986).

Brook, J.R., T.F. Dann y R.T. Burnett, The relationship among TSP, $P M_{10}, P M_{2,5}$ and inorganic constituents of atmospheric particulate mater at multiple Canadian locations. Journal of the Air \& Waste Management Association 47, 2-19 (1997).

Castanho, A. D. y P. Artaxo, Wintertime and summertime São Paulo aerosol source apportionment study. Atm. Environment 35, 4889-4902 (2001).

Celis, J., R. Morales, C. Zaror, J. Inzunza, R. Flocchini y O. Carvacho, Chemical characterization of the inhalable particulate matter in the city of Chillán, Chile. Journal of the Chilean Chemical Society 48, 47-53 (2003).

Chow, J. y J. Watson, Ion chromatography. In Elemental analysis of airborne particles. pp 97-137, Gordon and Breach Newark, NJ. (1999).

Chow, J., J. Watson, D. Lowenthal, P. Solomon, K. Magliano, S. Ziman y L. Richards, Temporal and spatial variations of PM2,5 and PM10 aerosol in the Southern California Air Quality Study. Atmospheric Environment 28, 2061-2080 (1994).

Kavouras, I., P. Koutrakis, F. Cereceda-Balic y P. Oyola, Source apportionment of $P M_{10}$ and $P M_{2,5}$ in five Chilean cities using factor analysis, Journal of Air Waste Management Association 51, 451-464 (2001). 
Kim, B., S. Teffera y M. Zeldin, Characterization of $P M_{2.5}$ and $P M_{10}$ in the south coast air basin of southern California: Part 1-Spatial variations, Journal of the Air \& Waste Management Association 50, 2034-2044 (2000).

Olave, D., Calidad de Vida en la Ciudad de Chillán-Chile. Revista Tiempo y Espacio 6, $61-73$ (1996).

Parkhurst, W., R. Tanner, F. Weatherford y R. Valente, Historic PM2.5/PM10 concentrations in the Southeastern United Stated-Potential implications of the revised particulate matter standard, Journal of the Air \& Waste Management Association 47, 1060-1067 (1999).

PLADECO. Plan comunal de desarrollo 1998-2000. Municipalidad de Chillán, Dirección de Planificación, Chillán (1998).

Préndez, M. y P. Ulriksen, La contaminación del aire y sus efectos, In Contaminación Atmosférica de Santiago: Estado Actual y Soluciones, pp 23-36 Universidad de Chile, Santiago, Chile (1993).

Querol, X., A. Alastuey, S. Rodríguez, F. Plana, E. Mantilla y C. Ruiz, Monitoring of $P M_{10}$ and $P M_{2.5}$ around primary particulate anthropogenic emission sources, Atmospheric Environment 35, 845-858 (2001).

Romieu, I. y V. Borja-Aburto, Particulate air pollution and daily mortality: can results be generalized to Latin American countries?, Salud Pública México 39, 403-411 (1997).

Röösli, M., G. Theis, N. Künzli, J. Staehelin, P. Mathys, L. Oglesby, M. Camenzind y Ch. BraunFahrländer, Temporal and spatial variation of the chemical composition of $P M_{10}$ at urban and rural sites in the Basel area, Switzerland, Atmospheric Environment 35, 3701-3713 (2001).

Salinas, F., Estudio experimental del efecto de isla calórica en la ciudad de Santiago, Memoria para optar al Título de ingeniero civil, Facultad de Ciencias Físicas y Matemáticas, Universidad de Chile, Santiago (1982).

Sánchez, J. y J. Morel, Una estimación de los beneficios en salud de reducir la contaminación en Santiago, In Economía del MedioAmbiente en América Latina, pp 157-178 Ediciones Universidad Católica, Santiago, Chile (1995).

Sandoval, H., Aire, In Informe País: Estado del Medio Ambiente en Chile, pp 35-73 Centro de Análisis de Políticas Públicas, Universidad de Chile, LOM Ediciones, Santiago, Chile (1999).

Tsapakis, M., E. Lagoudaki, E. Stephanou, I. Kavouras, P. Koutrakis, P. Oyola y D. Von Baer, The composition and sources of PM2.5 organic aerosol in two urban areas of Chile, Atmospheric Environment 36, 3851-3863 (2002).

Ulriksen, P., Factores meteorológicos de la contaminación atmosférica de Santiago, In Contaminación Atmosférica de Santiago: Estado Actual y Soluciones, pp 37-60 Universidad de Chile, Santiago, Chile (1993).

Viidanoja, J., M. Sillampää, J. Laakia, V-M. Kerminen, R. Hillamo, P. Aarnio y T. Koskentalo, Organic and black carbon in PM2.5 and PM10, Atmospheric Environment 36, 3183-3193 (2002). 